\title{
EXTRAPOLATION TO THE LIMIT BY USING CONTINUED FRACTION INTERPOLATION
}

\section{LUC WUYTACK}

1. The extrapolation problem. Assume that a convergent sequence $\left\{a_{0}, a_{1}, a_{2}, \cdots\right\}$ of real numbers is given with $A$ as limit. In order to find the limit $A$ numerically one can form a new sequence $\left\{b_{i}\right\}$, which has also $A$ as limit and whose convergence is faster. One way to perform the determination of $\left\{b_{i}\right\}$ is to use extrapolation methods.

Let $\left\{x_{0}, x_{1}, \cdots\right\}$ be a convergent sequence of points with $z$ as limit. The essential idea in extrapolation is to define a sequence of interpolating functions $\left\{y_{0}(x), y_{1}(x), \cdots\right\}$ such that $y_{n}\left(x_{i}\right)=a_{i}$ for $i=0,1$, $\cdots n$ and $n=0,1,2, \cdots$. The elements $b_{i}$ can be defined as follows $b_{i}=\lim _{x \rightarrow z} y_{i}(x)$ for $i=0,1,2, \cdots$, if these limits exist and are finite. The points $x_{i}$ are called interpolation points and $z$ is called the extrapolation point.

Let $R(\ell, m)$ be the class of ordinary rational functions $r_{\ell, m}=p / q$ where the degree of $p$ is at most $\ell$ and the degree of $q$ at most $m$. Under certain conditions it is possible to construct a set of rational functions $r_{\ell, m}$ for $\ell, m=0,1,2, \cdots$, satisfying $r_{\ell, m}\left(x_{i}\right)=a_{i}$ for $i=0$, $1, \cdots, \ell+m$. This set of functions can be arranged in a table as follows

$\begin{array}{ccccc}r_{0,0} & r_{0,1} & r_{0,2} & r_{0,3} & - \\ r_{1,0} & r_{1,1} & r_{1,2} & r_{1,3} & - \\ r_{2,0} & r_{2,1} & r_{2,2} & r_{2,3} & -\end{array}$

In the method of Neville (polynomial extrapolation) the first column is constructed. In the method of Bulirsch and Stoer (rational extrapolation) the "staircase" $r_{0,0}, r_{1,0}, r_{1,1}, r_{2,1}, \cdots$ is constructed. In both methods $z=0$ is used as extrapolation point and this makes the calculation of $b_{\ell+m}=r_{\ell, m}(z)$ very simple.

The elements $r_{0,0}, r_{1,1}, r_{2,2}, \cdots$ can be found by using Thiele's method for continued fraction interpolation. If $z=\infty$ is taken as extrapolation point then the values of $b_{i}$ can be computed by using a method similar to the $\epsilon$-algorithm (see [1], p. 186 and [2]).

This work was supported in part by the NFWO (Belgium) and a NATO-Fellowship. 
The effectiveness of the above methods depends highly on the convergence properties of $\left\{a_{i}\right\}$ and the choice of the interpolation points $x_{i}$. If very little is known about the convergence of $\left\{a_{i}\right\}$ it might be of interest to construct the whole table. A method for constructing the lower half of the table is given in the next section. A similar method can be used to get the upper half of the table.

2. An algorithm for rational interpolation. Consider the following continued fraction

$$
\begin{aligned}
f_{k}(x)= & c_{0} \cdot p_{0}(x)+c_{1} \cdot p_{1}(x)+\cdots c_{k-1} \cdot p_{k-1}(x) \\
& +\frac{c_{k} \cdot p_{k}(x)}{1}-\frac{q_{1}{ }^{k}\left(x-x_{k}\right)}{1} \\
& \quad-\frac{e_{1}^{k}\left(x-x_{k+1}\right)}{1}-\frac{q_{2}^{k}\left(x-x_{k+2}\right)}{1}-\frac{e_{2}^{k}\left(x-x_{k+3}\right)}{1}-\cdots
\end{aligned}
$$

where $p_{0}(x)=1$ and $p_{k}(x)=\left(x-x_{k-1}\right) \cdot p_{k-1}(x)$ for $k \geqq 1$. Under certain conditions the coefficients in this continued fraction can be defined such that the $n$th convergent $f_{k, n}$ of $f_{k}$ satisfies $f_{k, n}\left(x_{i}\right)=a_{i}$ for $i=0,1, \cdots, k-1+n$. Using contraction it is possible to obtain a continued fraction $f_{k}{ }^{\prime}$ whose convergents $f_{k, n}^{\prime}=P_{k, n} / Q_{k, n}$ satisfy the relation $f_{k, n}^{\prime}=f_{k, 2 n+1}$ for $n=0,1, \cdots$. If we also consider $f_{k+1}$ and define a continued fraction $f_{k+1}^{\prime}$ whose convergents satisfy $f_{k+1, n}^{\prime}=f_{k+1,2 n}$ then we have $f_{k, n}^{\prime}\left(x_{i}\right)=f_{k+1, n}^{\prime}\left(x_{i}\right)=a_{i}$ for $i=0$, $1, \cdots, k+2 n$. This means that there exists a nonzero constant $d_{n}{ }^{k}$ satisfying $P_{k+1, n}=d_{n}{ }^{k} . \quad P_{k, n}$ and $Q_{k+1, n}=d_{n}{ }^{k} \cdot Q_{k, n}$. The coefficients $c_{i}$ in $f_{k}$ and $f_{k+1}$ can be obtained by using divided differences. In order to get the other coefficients the following recurrence relations can be used. The starting values are

$$
\begin{aligned}
& d_{1}{ }^{k}=1+\frac{c_{k+1}}{c_{k}} \cdot\left[x_{k+1}-x_{k}\right] ; \\
& q_{1}{ }^{k}=\frac{1}{d_{1}{ }^{k}} \cdot \frac{c_{k+1}}{c_{k}} ; \quad e_{1}{ }^{k}=\frac{1}{d_{1}{ }^{k}} \cdot q_{1}{ }^{k+1}-q_{1}{ }^{k} .
\end{aligned}
$$

For $i \geqq 2$ we have, with $y_{k, i}=x_{k+2 i-2}-x_{k+2 i-1}$,

$$
\begin{aligned}
& d_{i}^{k}=d_{i-1}^{k} \cdot\left(1+y_{k, i} \cdot e_{i-1}^{k+1}\right)-d_{i-2}^{k} \cdot y_{k, i} \cdot q_{i-1}^{k+1} \cdot \frac{e_{i-1}^{k+1}}{e_{i-1}^{k}} ; \\
& q_{i}{ }^{k}=\frac{d_{i-2}^{k}}{d_{i}{ }^{k}}\left(d_{i-1}^{k+1} \cdot \frac{e_{i-1}^{k+1}}{e_{i-1}^{k}}\right) ; \quad e_{i}{ }^{k}=\frac{d_{i-1}^{k}}{d_{i}{ }^{k}}\left(e_{i-1}^{k+1}+q_{i}{ }^{k+1}\right)-q_{i}{ }^{k} .
\end{aligned}
$$


This algorithm is similar to the $q d$-algorithm and much of the research done for the $q d$-algorithm can also be done for the above algorithm. The convergents of $f_{k}$ for $k=1,3,5, \cdots$, form the lower half of the table.

\section{REFERENCES}

1. F. M. Larkin, Some techniques for rational interpolation, The Computer Journal 10 (1967), 178-187. MR 35 \#6334.

2. L. Wuytack, A new technique for rational extrapolation to the limit, Numerische Mathematik 17 (1971), 215-221.

UNiversity OF ANTWERP, B-2610 WiLriJK, (BELgiUm) 
, 